\title{
CRITICAL CONVECTIVE-TYPE EQUATIONS ON A HALF-LINE
}

\author{
ELENA I. KAIKINA
}

Received 16 June 2005; Revised 6 October 2005; Accepted 4 January 2006

We are interested in the global existence and large-time behavior of solutions to the initial-boundary value problem for critical convective-type dissipative equations $u_{t}+$ $\mathbb{N}\left(u, u_{x}\right)+\left(a_{n} \partial_{x}^{n}+a_{m} \partial_{x}^{m}\right) u=0,(x, t) \in \mathbb{R}^{+} \times \mathbb{R}^{+}, u(x, 0)=u_{0}(x), x \in \mathbb{R}^{+}, \partial_{x}^{j-1} u(0, t)=0$ for $j=1, \ldots, m / 2$, where the constants $a_{n}, a_{m} \in \mathbb{R}, n, m$ are integers, the nonlinear term $\mathbb{N}\left(u, u_{x}\right)$ depends on the unknown function $u$ and its derivative $u_{x}$ and satisfies the estimate $|\mathbb{N}(u, v)| \leq C|u|^{\rho}|v|^{\sigma}$ with $\sigma \geq 0, \rho \geq 1$, such that $((n+2) / 2 n)(\sigma+\rho-1)=1, \rho \geq 1$, $\sigma \in[0, m)$. Also we suppose that $\int_{\mathbb{R}^{+}} x^{n / 2} \mathbb{N} d x=0$. The aim of this paper is to prove the global existence of solutions to the inital-boundary value problem above-mentioned. We find the main term of the asymptotic representation of solutions in critical case. Also we give some general approach to obtain global existence of solution of initial-boundary value problem in critical convective case and elaborate general sufficient conditions to obtain asymptotic expansion of solution.

Copyright @ 2006 Hindawi Publishing Corporation. All rights reserved.

\section{Introduction}

We consider the initial-boundary value problem on a half-line for critical nonlinear dissipative equations of convective type:

$$
\begin{gathered}
u_{t}+\mathbb{N}\left(u, u_{x}\right)+\mathbb{K}(u)=0, \quad t>0, x>0, \\
\partial_{x}^{j-1} u(0, t)=0, \quad t>0, j=1, \ldots, M, \\
u(x, 0)=u_{0}(x), \quad x>0 .
\end{gathered}
$$

The linear part of (1.1) is a differential operator

$$
\mathbb{K}(u)=a_{n} \partial_{x}^{n}+a_{m} \partial_{x}^{m},
$$

where $a_{n}, a_{m} \in \mathbb{R}, n, m$ are integers, $m>n$. Note that the operator $\mathbb{K}(u)$ has the 
symbol

$$
K(p)=a_{n} p^{n}+a_{m} p^{m}
$$

In this paper, we suppose that the dissipation condition is fulfilled:

$$
\operatorname{Re} K(p)>0 \quad \text { for } \operatorname{Re} p=0, p \neq 0 .
$$

We assume that the nonlinearity $\mathbb{N}\left(u, u_{x}\right)$ satisfies the estimate

$$
|\mathbb{N}(u, v)| \leq C|u|^{\rho}|v|^{\sigma}
$$

with $\rho, \sigma \geq 0$ and is of convective type such that

$$
\int_{0}^{+\infty} x^{n / 2} \mathbb{N}\left(u, u_{x}\right) d x=0
$$

and for any $q \in \mathbb{C}$,

$$
\mathbb{N}(v q)=q^{\sigma+\rho} \mathbb{N}(v) .
$$

The number of the boundary data $M=m / 2$ ( or $M=(m+1) / 2$ in the case of odd integer $m$ and $a_{m}<0$ ) (see book [7]). Equation (1.1) is a simple universal model, which appears as the first approximation in the description of the dispersive dissipative nonlinear waves (see [14]).

The global existence and large-time asymptotic behavior of solutions to the Cauchy problems for nonlinear local and nonlocal equations were studied in many works (see $[1-6,8,9,12,13,15]$ and literature cited therein).

In papers $[10,11]$,the large-time asymptotic behavior of solutions to the Korteweg-de Vries-Burgers-type equations on a half-line in the super critical case was found. A general theory of nonlinear nonlocal homogeneous equations on a half-line was developed in book [7]. In the present paper, we consider (1.1) in the case of operator $\mathbb{K}$ with nonhomogeneous symbol $K(p)$. As a typical example, we take a polynomial $K(p)=a_{n} p^{n}+a_{m} p^{m}$. We are interested in the global existence and large-time behavior of solutions to the initial-boundary value problem (1.1) in the critical convective-type case, when the nonlinear term of equation has the same time decay rate as that of the linear terms and has zero moment. Also in this paper, we give some general approach to obtain global existence of solution of initial-boundary value problem in critical convective case. We elaborate general sufficient conditions to obtain asymptotic expansion of solution.

We denote the usual Lebesgue space as $\mathbf{L}^{p}$ on $\mathbb{R}^{+}$by the norm $\|\phi\|_{\mathbf{L}^{p}}=\left(\int_{\mathbb{R}^{+}}|\phi(x)| p d x\right)^{1 / p}$, and the weighted Lebesgue space as $\mathbf{L}^{p, a}, a \geq 0$, with norm

$$
\|\phi\|_{\mathrm{L}^{p, a}}=\left\|\langle\cdot\rangle^{a} \phi\right\|_{\mathbf{L}^{p}}, \quad\langle x\rangle=\sqrt{1+|x|^{2}} .
$$

Also we introduce the weighted Sobolev space

$$
\mathbf{W}_{p}^{k, a}=\left\{\phi \in \mathbf{L}^{p, a}, \sum_{j=0}^{k}\left\|\partial_{x}^{j} \phi\right\|_{\mathbf{L}^{p, a}}<\infty\right\} .
$$


By $\mathbf{C}(\mathbf{I} ; \mathbf{B})$ we denote the space of continuous functions from a time interval $\mathbf{I}$ to the Banach space $\mathbf{B}$. We denote $\{x\}=x /\langle x\rangle$.

We assume that the initial data $u_{0} \in \mathbf{L}^{\infty} \cap \mathbf{L}^{1, n / 2+1}$ and satisfies condition

$$
\left\|u_{0}\right\|_{\mathbf{L}^{\infty}}+\left\|u_{0}\right\|_{\mathbf{L}^{1, n / 2+1}}<\mathcal{\varepsilon}
$$

where $\varepsilon>0$ is sufficiently small. We assume that the nonlinear term $\mathbb{N}\left(u, u_{x}\right)$ is of critical convective type from the point of view of the large-time asymptotic behavior, that is, the orders $\sigma$ and $\rho$ of the nonlinear term satisfy the following condition:

$$
\begin{gathered}
\frac{n+2}{2 n}(\sigma+\rho-1)=1, \quad \rho \geq 1, \sigma \in[0, m), \\
\int_{\mathbb{R}^{+}} x^{n / 2} \mathbb{N} d x=0 .
\end{gathered}
$$

We denote

$$
\begin{aligned}
G_{1}(s, q) & =\frac{1}{2 \pi i} \int_{-i \infty}^{i \infty} e^{-a_{n} z^{n}}\left(e^{z(s-q)}-\sum_{j=1}^{n / 2} A_{j} e^{-\left(s+r_{j} q\right)}\right) d z, \\
A_{j} & =\prod_{k=1, k \neq j}^{n / 2} \frac{1+r_{k}}{1-r_{k}}, \quad a_{n} r_{k}^{n}=-1, \operatorname{Re} r_{k}>0 .
\end{aligned}
$$

Our purpose in this paper is to prove the following result.

Theorem 1.1. Assume that the conditions (1.10)-(1.11) are valid. Then there exists a unique solution

$$
u(t, x) \in \mathbf{C}\left([0, \infty) ; \mathbf{L}^{\infty} \cap \mathbf{L}^{1, n / 2+1}\right) \cap \mathbf{C}\left((0, \infty) ; \mathbf{W}_{\infty}^{1,0} \cap \mathbf{W}_{1}^{1, n / 2+1}\right)
$$

of the initial-boundary value problem (1.1). Furthermore, there exists a function $V \in \mathbf{L}^{\infty}$ such that the asymptotic formula

$$
\left\|\langle t\rangle^{\gamma}\left(u(t)-t^{-(n+2) / 2 n} V\left((\cdot) t^{-1 / n}\right)\right)\right\|_{\mathbf{L}^{\infty}} \leq C \varepsilon
$$

is valid for $t \rightarrow \infty$ uniformly with respect to $x \in \mathbb{R}^{+}$, where $Q=n / 2, \gamma=((1 / 2) \mu, 1-\sigma / m)$, and $V(\xi)$ is the solution of the integral equation

$$
\begin{aligned}
V(\xi)=f\left(u_{0}\right) G_{0}(\xi)-\frac{1}{\beta} \int_{0}^{1} & \frac{d z}{z^{((n+2) / 2 n)(\rho+\sigma)}(1-z)^{1 / n}} \\
& \times \int_{\mathbb{R}^{+}} G_{1}\left(\frac{\xi}{(1-z)^{1 / n}}, \frac{y z^{1 / n}}{(1-z)^{1 / n}}\right) \mathbb{N}(V(y)) d y,
\end{aligned}
$$

where

$$
f\left(u_{0}\right)=\int_{\mathbb{R}^{+}} x^{Q} u_{0} d x
$$


We organize the rest of our paper as follows. In Section 2, we give general theory of studying initial-boundary value problem in the case of critical convective type of nonlinearity. In Sections 3 and 4, we construct the Green function of the solution of the linear problem. We prove preliminary lemmas in Section 5. In Lemma 5.1, we obtain estimates of the Green operator in the Lebesgue spaces $\mathbf{L}^{r}, 1 \leq r \leq \infty$, and $\mathbf{L}^{1, Q+1}$. Then in Lemma 5.3, we estimate the Green operator in our basic norm

$$
\begin{array}{r}
\|\phi\|_{\mathbf{X}}=\sup _{t>0}\left(\sum _ { k = 0 } ^ { 1 } \left(\{t\}^{k / m}\langle t\rangle^{(Q+k) / n+(1 / n)(1-1 / r)}\left\|\partial_{x}^{k} \phi(t)\right\|_{\mathbf{L}^{r}}\right.\right. \\
\left.\left.+\{t\}^{k / m}\langle t\rangle^{(k-1) / n}\left\|\partial_{x}^{k} \phi(t)\right\|_{\mathbf{L}^{1, Q+1}}\right)\right) .
\end{array}
$$

\section{General approach}

Suppose we can rewrite the initial-boundary value problem (1.1) as the following integral equation:

$$
u(t)=\mathscr{G}(t) u_{0}-\int_{0}^{t} \mathscr{G}(t-\tau) \mathcal{N}(u(\tau)) d \tau
$$

where $\mathscr{G}$ is the Green operator of the corresponding linear problem. Here $\mathcal{N}(u)$ is some nonlinear operator such that for any $q \in \mathbb{C}$,

$$
\mathcal{N}(v q)=q^{\sigma_{1}+1} \mathcal{N}(v)
$$

In this section, we prove global-in-time existence of solutions to the problem (1.1). By the global solution of the problem (1.1), we always understand the solution $u(t)$ of the corresponding integral equation (2.1), belonging to some complete metric space $\mathbf{X}$ of functions defined on $[0, \infty) \times \mathbb{R}^{+}$(the so-called mild solution).

We fix a metric space $\mathbf{Z}$ of functions defined on $\mathbb{R}^{+}$.

We suppose that there exists the asymptotic kernel $\widetilde{G_{0}}(x, t)=t^{-\alpha} G_{0}\left(x t^{-1 / n}\right) \in \mathbf{X}$ in spaces $\mathbf{X}, \mathbf{Z}$, such that

$$
\left\|\langle t\rangle^{\gamma}\left(\mathscr{G}(t) \phi-G_{0} f(\phi)\right)\right\|_{\mathbf{X}} \leq C\|\phi\|_{\mathbf{Z}}
$$

with some $\alpha>0, n>0$, where $f(\phi)$ is the linear functional: $\mathbf{Z} \rightarrow \mathbb{R}$ and

$$
f\left(t^{-\alpha} \phi\left((\cdot) t^{-1 / n}\right)\right)=f(\phi)
$$

for all $t>0, \phi \in \mathbf{Z}$.

We also fix a metric space $\mathbf{W}$ of functions defined on $\mathbb{R}^{+}$, such that the norm of $\mathbf{W}$ is induced by the norm of $\mathbf{X}$ by the relation

$$
\|\phi\|_{\mathrm{W}}=\left\|t^{-\alpha} \phi\left((\cdot) t^{-1 / n}\right)\right\|_{\mathbf{X}}
$$


Theorem 2.1. Assume that the initial data $u_{0} \in \mathbf{Z}$, and it is such that $\left\|u_{0}\right\|_{\mathrm{Z}}<\varepsilon$. Let there exist function $G_{1}(x, y)\left(G_{1}(x, 0)=0\right)$ such that for operator

$$
\mathscr{G}_{0}(t) \phi=t^{-\beta} \int_{\mathbb{R}^{+}} G_{1}\left(x t^{-1 / n}, y t^{-1 / n}\right) \phi(y) d y,
$$

the following estimates with $\gamma>0$ exist:

$$
\left\|\mathscr{G}_{0}(t) \phi\right\|_{\mathrm{X}}+\left\|\langle t\rangle^{\gamma}\left(\mathscr{G}(t)-\mathscr{G}_{0}(t)\right) \phi\right\|_{\mathrm{X}} \leq C\|\phi\|_{\mathrm{Z}}
$$

and if $f(\phi)=0$,

$$
\begin{gathered}
\left\|\int_{0}^{t} \mathscr{G}_{0}(t-\tau) \phi(\tau) d \tau\right\|_{\mathbf{X}} \leq C\|\phi(t)\|_{\mathbf{X}}, \\
\left\|\langle t\rangle^{\gamma} \int_{0}^{t}\left(\varphi(t-\tau)-\varphi_{0}(t-\tau)\right) \phi(\tau) d \tau\right\|_{\mathbf{X}} \leq C\|\phi(t)\|_{\mathbf{X}} .
\end{gathered}
$$

Also, suppose that the nonlinearity $\mathcal{N}$ in (1.1) is a critical convective such that $f(\mathcal{N}(u))=0$ for any $u \in \mathbf{X}$,

$$
\begin{gathered}
\left\|\left(\mathcal{N}\left(u_{1}\right)-\mathcal{N}\left(u_{2}\right)\right)\right\|_{\mathbf{X}} \leq C\left\|\left(u_{1}-u_{2}\right)\right\|_{\mathbf{X}}\left(\left\|u_{1}\right\|_{\mathbf{X}}^{\sigma_{1}}+\left\|u_{2}\right\|_{\mathbf{X}}^{\sigma_{1}}\right), \\
\sigma_{1} \alpha+\beta=1+\frac{1}{n} .
\end{gathered}
$$

Then there exists a unique global solution $u \in \mathbf{C}([0, \infty) ; \mathbf{X})$ of the initial-boundary value problem (1.1). Furthermore, there exists a function $V \in \mathbf{W}$ such that the asymptotic formula

$$
\left\|\langle t\rangle^{\gamma}\left(u(t)-t^{-\alpha} V\left((\cdot) t^{-1 / n}\right)\right)\right\|_{\mathbf{X}} \leq C \varepsilon
$$

is valid for $t \rightarrow \infty$ uniformly with respect to $x \in \mathbb{R}^{+}$, where $V(\xi)$ is the solution of the integral equation

$$
V(\xi)=f\left(u_{0}\right) G_{0}(\xi)-\int_{0}^{1} \mathscr{G}_{0}(1-z) \mathcal{N}\left(z^{-\alpha} V\left((\cdot) z^{-1 / n}\right)\right) d z
$$

Proof. We prove the existence of the solution $u(x, t)$ for the initial-boundary value problem $(1.1)$ by the successive approximations $u_{m}(x, t), m=1,2, \ldots$, defined as follows:

$$
\partial_{t} u_{m}+\mathbb{K}\left(u_{m}\right)=\mathcal{N}\left(u_{m-1}\right), \quad v_{m}(0, x)=v_{0}(x),
$$

for all $m \geq 2$, where

$$
u_{1}=\varphi_{0}(t) u_{0}
$$

The integral equation associated with (1.1) is written as

$$
u_{m}(t)=\mathscr{G}(t) u_{0}-\int_{0}^{t} \mathscr{G}(t-\tau) \mathcal{N}\left(u_{m-1}\right) d \tau
$$


We now prove by induction the following estimates for all $m \geq 1$ :

$$
\left\|u_{m}\right\|_{\mathbf{X}} \leq C \varepsilon, \quad\left\|u_{m}(t)-\mathscr{G}(t) u_{0}\right\|_{\mathbf{X}} \leq C \varepsilon
$$

From (2.7), we have

$$
\left\|\mathscr{G}_{0}(t) u_{0}\right\|_{\mathrm{X}} \leq C \varepsilon, \quad\left\|u_{1}-\mathscr{G}(t) u_{0}\right\|_{\mathrm{X}} \leq C \varepsilon
$$

Therefore estimates (2.17) are valid for $m=1$. We assume that estimates (2.17) are true with $m$ replaced by $m-1$. Due to (2.10), we have

$$
\|\mathcal{N}(u)\|_{\mathbf{X}} \leq C\|u\|_{\mathbf{X}}^{\sigma_{1}+1} .
$$

Since $x^{Q \mathcal{N}}\left(u_{m-1}(\tau)\right)$ have the zero-mean value via (2.8) and (2.9), we get

$$
\begin{aligned}
\left\|\int_{0}^{t} \mathscr{G}(t-\tau) \mathcal{N}\left(u_{m-1}(\tau)\right) d \tau\right\|_{\mathbf{X}} \leq & \left\|\int_{0}^{t} \mathscr{G}_{0}(t-\tau) \mathcal{N}\left(u_{m-1}(\tau)\right) d \tau\right\|_{\mathbf{X}} \\
& +\left\|\int_{0}^{t}\left(\mathscr{G}(t-\tau)-\mathscr{G}_{0}(t-\tau)\right) \mathcal{N}\left(u_{m-1}(\tau)\right) d \tau\right\|_{\mathbf{X}} \\
\leq & \left\|\mathcal{N}\left(u_{m-1}\right)\right\|_{\mathbf{X}} \leq C \varepsilon .
\end{aligned}
$$

It follows that

$$
\left\|u_{m}\right\|_{\mathbf{X}} \leq C \varepsilon,\left\|u_{m}(t)-\mathscr{G}(t) u_{0}\right\|_{\mathbf{X}} \leq C \varepsilon
$$

Thus by induction, we see that estimates (2.17) are valid for all $m \geq 1$. In the same way by induction, we can prove that

$$
\left\|u_{m}-u_{m-1}\right\|_{\mathbf{X}} \leq \frac{1}{4}\left\|u_{m-1}-u_{m-2}\right\|_{\mathbf{X}}
$$

for all $m>2$. Therefore taking the limit $m \rightarrow \infty$, we obtain a unique solution $\lim _{m \rightarrow \infty} u_{m}(t$, $x)=u(t, x) \in \mathbf{X}$, satisfying the equation

$$
u(t)=\mathscr{G}(t) u_{0}-\int_{0}^{t} \mathscr{G}(t-\tau) \mathcal{N}(u) d \tau
$$

and estimates for $t>1$,

$$
\left\|u(t)-\varphi(t) u_{0}\right\|_{\mathbf{X}} \leq C \varepsilon, \quad\|u(t)\|_{\mathbf{X}} \leq C \varepsilon .
$$

We now compute the asymptotics of the solution. First, we show the existence of solutions to the integral equation

$$
V(\xi)=V_{0}(\xi)-\int_{0}^{1} \varphi_{0}(1-z) \mathcal{N}\left(z^{-\alpha} V\left((\cdot) z^{-1 / n}\right)\right) d z
$$


where $V_{0}(\xi)=\theta G_{0}(\xi)$, where

$$
\theta=f\left(u_{0}\right)
$$

We prove the existence of the solution $V$ for integral equation (2.25) by the contraction mapping principle. We define the transformation $\mathscr{R}(V)$ by the formula

$$
\mathscr{R}(V)=\theta G_{0}-\int_{0}^{1} \mathscr{G}_{0}(1-z) \mathcal{N}\left(z^{-\alpha} V\left((\cdot) z^{-1 / n}\right)\right) d z
$$

for any $V \in \mathbf{W}_{\rho}$, where

$$
\mathbf{W}_{\rho}=\left\{V \in \mathbf{W}:\|V\|_{\mathbf{W}} \leq \rho\right\}
$$

and $C|\theta| \leq \rho$ and $\rho>0$ sufficiently small. First we check that the mapping $\mathscr{R}$ transforms the set $\mathbf{W}_{\rho}$ into itself. By the property (2.34) using relation of the norms $\mathbf{W}$ and $\mathbf{X}$, we have for $v(t, x)=t^{-\alpha} V\left(x t^{-1 / n}\right)$,

$$
\begin{aligned}
\|\mathscr{R}(V)\|_{\mathrm{W}} & \leq\left\|\theta G_{0}\right\|_{\mathrm{W}}+\left\|\int_{0}^{1} \mathscr{G}_{0}(1-z, \xi) \mathcal{N}\left(z^{-\alpha} V\left((\cdot) z^{-1 / n}\right)\right) d z\right\|_{\mathrm{W}} \\
& \leq C|\theta|+\left\|t^{\alpha} \int_{0}^{t} \mathscr{G}_{0}\left(t-\tau, \xi t^{1 / n}\right) \mathcal{N}(v(\tau)) d \tau\right\|_{\mathrm{W}} \\
& =C|\theta|+\left\|\int_{0}^{t} \mathscr{G}_{0}(t-\tau) \mathcal{N}(v(\tau)) d \tau\right\|_{\mathbf{X}}
\end{aligned}
$$

Then due to (2.8), we get

$$
\left\|\int_{0}^{t} G_{0}(t-\tau) \mathcal{N}(v(\tau)) d \tau\right\|_{\mathbf{X}} \leq C\|v\|_{\mathbf{X}}^{\sigma_{1}+1}=C\|V\|_{\mathbf{Q}}^{\sigma_{1}+1} \leq C \rho^{\sigma_{1}+1} .
$$

Hence $\|\mathscr{R}(V)\|_{\mathrm{W}} \leq C|\theta|+C \rho^{\sigma_{1}+1} \leq C \rho$ if $\rho>0$ is small. In the same manner, we estimate the difference, denoting that $w(t, x)=t^{-\alpha} W\left(x t^{-1 / n}\right)$ and using the relation of the norms $\mathbf{W}$ and $\mathbf{X}$,

$$
\begin{aligned}
\|\mathscr{R}(V)-\mathscr{R}(W)\|_{\mathrm{W}} & \leq\left\|\int_{0}^{1} \mathscr{G}_{0}(1-z, \xi)\left(\mathcal{N}\left(z^{-\alpha} V\left((\cdot) z^{-1 / n}\right)\right)-\mathcal{N}\left(z^{-\alpha} W\left((\cdot) z^{-1 / n}\right)\right)\right) d z\right\|_{\mathrm{W}} \\
& =\left\|\int_{0}^{t} \mathscr{G}_{0}(t-\tau)(\mathcal{N}(v(\tau))-\mathcal{N}(w(\tau))) d \tau\right\|_{\mathbf{X}} \\
& \leq \frac{1}{2}\|V-W\|_{\mathrm{W}}\left(\|V\|_{\mathrm{W}}^{\sigma_{1}}+\|W\|_{\mathrm{W}}^{\sigma_{1}}\right) \leq C \rho^{\sigma_{1}}\|V-W\|_{\mathrm{W}} \leq \frac{1}{2}\|V-W\|_{\mathrm{W}} .
\end{aligned}
$$

Therefore, $\mathscr{R}$ is a contraction mapping in the closed set $\mathbf{W}_{\rho}$ of a complete metric space X. Hence, there exists a unique solution $V \in \mathbf{W}_{\rho}$ to the integral equation (2.25). We now prove that self-similar solutions $V(\xi)$ give us the asymptotics of solutions to the problem 
(1.1). Denote $v(t)=t^{-\alpha} \theta V\left((\cdot) t^{-1 / n}\right)$, where $V$ satisfies integral equation (2.25), then we have

$$
v(t)=\theta t^{-\alpha} G_{0}\left((\cdot) t^{-1 / n}\right)-\int_{0}^{t} \varphi_{0}(t-\tau) \mathcal{N}(v(\tau)) d \tau .
$$

Since $u(t)$ satisfies the integral equation (2.1),

$$
u(t)=\mathscr{G}(t) u_{0}-\int_{0}^{t} \mathscr{G}(t-\tau) \mathcal{N}(u(\tau)) d \tau,
$$

we write for the difference

$$
\begin{aligned}
\left\|\langle t\rangle^{\gamma}(u(t)-v(t))\right\|_{\mathbf{X}} \leq & \left\|\langle t\rangle^{\gamma}\left(\mathscr{G}(t) u_{0}-\theta t^{-\alpha} G_{0}\left((\cdot) t^{-1 / n}\right)\right)\right\|_{\mathbf{X}} \\
& +\left\|\langle t\rangle^{\gamma} \int_{0}^{t} \mathscr{G}(t-\tau)(\mathcal{N}(u(\tau))-\mathcal{N}(v(\tau))) d \tau\right\|_{\mathbf{X}} \\
& +\left\|\langle t\rangle^{\gamma} \int_{0}^{t}\left(\mathscr{G}(t-\tau)-\mathscr{G}_{0}(t-\tau)\right) \mathcal{N}(v(\tau)) d \tau\right\|_{\mathbf{X}} \\
\equiv & I_{1}+I_{2}+I_{3} .
\end{aligned}
$$

Note that in view of the relation of the norms $\mathbf{X}$ and $\mathbf{W}$, we have

$$
\|v\|_{\mathbf{X}}=|\theta|\|V\|_{\mathrm{W}} \leq C|\theta| \leq C \varepsilon .
$$

Then by the definition of the asymptotic kernel (see (2.3)), we obtain

$$
I_{1}=\left\|\langle t\rangle^{\gamma}\left(\mathscr{G}(t) u_{0}-\theta t^{-\alpha} G_{0}\left((\cdot) t^{-1 / n}\right)\right)\right\|_{\mathbf{X}} \leq C\left\|u_{0}\right\|_{\mathbf{Z}} \leq C \varepsilon .
$$

By virtue of condition (2.8), we get

$$
\begin{aligned}
I_{2} & =\left\|\langle t\rangle^{\gamma} \int_{0}^{t} \mathscr{G}(t-\tau)(\mathcal{N}(u(\tau))-\mathcal{N}(v(\tau))) d \tau\right\|_{\mathbf{X}} \\
& \leq C\left\|\langle t\rangle^{\gamma}(u(t)-v(t))\right\|_{\mathbf{X}}\left(\|u\|_{\mathbf{X}}^{\sigma_{1}}+\|v\|_{\mathbf{X}}^{\sigma_{1}}\right) \\
& \leq C \varepsilon^{\sigma}\left\|\langle t\rangle^{\gamma}(u(t)-v(t))\right\|_{\mathbf{X}}
\end{aligned}
$$

and via condition (2.9), we find

$$
\begin{aligned}
I_{3} & =\left\|\langle t\rangle^{\gamma} \int_{0}^{t}\left(\mathscr{G}(t-\tau)-\mathscr{G}_{0}(t-\tau)\right) \mathcal{N}(v(\tau)) d \tau\right\|_{\mathbf{X}} \\
& \leq C\|v\|_{\mathrm{X}}^{\sigma_{1}+1} \leq C\|V\|_{\mathrm{W}}^{\sigma_{1}+1} \leq C\|\theta\|^{\sigma+1} \leq C \varepsilon^{\sigma+1} .
\end{aligned}
$$

Now (2.34) implies that

$$
\left\|\langle t\rangle^{\gamma}(u(t)-v(t))\right\|_{\mathbf{X}} \leq C \varepsilon+C \varepsilon^{\sigma}\left\|\langle t\rangle^{\gamma}(u(t)-v(t))\right\|_{\mathbf{X}},
$$

hence estimate (2.12) follows since $\varepsilon>0$ is small enough. Theorem 2.1 is proved. 


\section{Linear problem}

We consider the linear initial-boundary value problem corresponding to (1.1):

$$
\begin{gathered}
u_{t}+\mathbb{K} u=f(x, t), \quad t>0, x>0, \\
u(x, 0)=u_{0}(x), \quad x>0, \\
\partial_{x}^{j-1} u(0, t)=h_{j}(t), \quad t>0, \text { for } j=0, \ldots, M .
\end{gathered}
$$

We define symbol of operator $\mathbb{K}$ as

$$
K(p)=a_{n} p^{n}+a_{m} p^{m}
$$

where $n, m$ are even integers, $n \leq m / 2+1, a_{n} \neq 0, a_{m} \neq 0$. We denote by $\phi_{j}(\xi)=K^{-1}(-\xi)$, $j=1, \ldots, N=m / 2$, different roots of equation for equation $K(p)=-\xi$, such that

$$
\operatorname{Re} \phi_{j}(\xi)>0
$$

for all $\xi \in D, \operatorname{Re} \xi>0$. Here $D$ is domain of analyticity of functions $\phi_{j}(\xi)$ with boundary

$$
\Gamma=\left\{(-i \infty,-i 0) \bigcup_{k}\left(\left[-i 0, K\left(p_{k}\right) e^{i 2 \pi}\right] \cup\left[K\left(p_{k}\right), i 0\right]\right) \cup(i 0, i \infty)\right\},
$$

where $K^{\prime}\left(p_{k}\right)=0$. Also we define the matrix

$$
\mathbb{A}=\left(\begin{array}{ccccc}
\phi_{1}^{N-1} & \phi_{1}^{N-2} & \phi_{1}^{N-3} & \cdots & 1 \\
\phi_{2}^{N-1} & \phi_{2}^{N-2} & \phi_{2}^{N-3} & \cdots & 1 \\
\vdots & \vdots & \vdots & \cdots & \vdots \\
\phi_{N}^{N-1} & \phi_{N}^{N-2} & \phi_{N}^{N-3} & \cdots & 1
\end{array}\right)
$$

and the vector

$$
\overrightarrow{\mathbf{B}}=\left(\begin{array}{c}
e^{-\phi_{1}(\xi) y} \\
e^{-\phi_{2}(\xi) y} \\
\cdots \\
e^{-\phi_{N}(\xi) y}
\end{array}\right) .
$$

In this section, we follow the method of the book [7] to obtain the explicit formula for the solution of the linear problem (3.1) under the condition

$$
u_{0} \in \mathbf{L}^{1}\left(\mathbb{R}^{+}\right), \quad f \in \mathbf{L}^{q}\left(0, T ; \mathbf{L}^{1}\left(\mathbb{R}^{+}\right)\right)
$$

with $q>2$. We have that solution of problem (3.1) has the following form:

$$
u(x, t)=\int_{0}^{+\infty} u_{0}(y) G(x, y, t) d y+\int_{0}^{t} d \tau \int_{0}^{+\infty} f(x, y, \tau) G(x, y, t-\tau) d y,
$$


where

$$
\begin{gathered}
G(x, y, t)=\frac{1}{2 \pi i} \int_{-i \infty}^{i \infty} e^{p x} H(p, y, t) d p \\
H(p, y, t)=e^{-p y-K(p) t}-\sum_{j=1}^{N} p^{N-j} \frac{1}{2 \pi i} \int_{\Gamma} \frac{e^{\xi t}}{K(p)+\xi}\left(\mathbb{A}^{-1} \overrightarrow{\mathbf{B}}\right)_{j} .
\end{gathered}
$$

Denote

$$
\tilde{H}\left(p, \phi_{1}, \phi_{2}, \ldots, \phi_{N}, y\right)=\sum_{k=1}^{N} p^{N-k}\left(\mathbb{A}^{-1} \vec{B}\right)_{k} .
$$

We write function $\tilde{H}$ in the form

$$
\tilde{H}=\sum_{k=1}^{N} p^{N-k} \sum_{j=1}^{N} e^{-\phi_{j} y}\left(\mathbb{A}^{-1} \vec{E}_{j}\right)_{k},
$$

where $\vec{E}_{j}$ is vector with component $e_{l}, l=1, \ldots, N$,

$$
e_{l}= \begin{cases}1, & l=j \\ 0, & l \neq j\end{cases}
$$

Using the results of book [7], we have

$$
\left(\mathbb{A}^{-1} \vec{E}_{j}\right)_{k}=(-1)^{k-1} \sigma_{k-1}\left(\phi_{1}, \phi_{2}, \ldots, \phi_{k-1}, \phi_{k+1}, \ldots, \phi_{N}\right) \prod_{l=1, l \neq k}\left(\phi_{k}-\phi_{l}\right)^{-1},
$$

where $\sigma_{k}$ are symmetrical polynomials, such that

$$
\begin{gathered}
\sigma_{0}\left(\phi_{2}, \ldots, \phi_{N}\right)=1, \\
\sigma_{1}\left(\phi_{2}, \ldots, \phi_{N}\right)=\sum_{j=2}^{N} \phi_{j}, \\
\sigma_{2}\left(\phi_{2}, \ldots, \phi_{N}\right)=\sum_{j \neq k} \phi_{j} \phi_{k}, \\
\vdots \\
\sigma_{N}\left(\phi_{2}, \ldots, \phi_{N}\right)=\prod_{j=2}^{N} \phi_{j} .
\end{gathered}
$$

Therefore from the Viett theorem, we obtain

$$
\tilde{H}=\sum_{j=1}^{N} \frac{e^{-\phi_{j} y} P_{N}(p)}{P_{N}^{\prime}\left(\phi_{j}\right)\left(p-\phi_{j}\right)},
$$


where by $P_{N}(p)$, we denote

$$
P_{N}(p)=\prod_{l=1}^{N}\left(p-\phi_{l}\right)
$$

We easily see that function $\tilde{H}\left(p, \phi_{1}, \phi_{2}, \ldots, \phi_{N}, y\right)$ is symmetrical with respect to variables $\phi_{j}(\xi)$, such that, for example,

$$
\tilde{H}\left(p, \phi_{1}, \phi_{2}, \ldots, \phi_{N}, y\right)=\tilde{H}\left(p, \phi_{2}, \phi_{1}, \ldots, \phi_{N}, y\right) .
$$

Since $\mathfrak{R} K(p)>0$ for all $\mathfrak{R} p=0, p \neq 0$ and the function $\tilde{H}\left(p, \phi_{1}, \ldots, \phi_{N}, y\right)$ is analytic in the domain $D$ and has the estimate

$$
\left|\tilde{H}\left(p, \phi_{1}, \ldots, \phi_{N}, y\right)\right| \leq C e^{-C y \sqrt[m]{|\xi|}}
$$

for $|\xi| \rightarrow \infty, \mathfrak{k}>0$ (see asymptotic formulas (4.2)), therefore by the Cauchy theorem and symmetrical properties of function $\tilde{H}$, we can change the contour of integration $\Gamma$ to the imaginary axis $(-i \infty, i \infty)$ to get

$$
\int_{\Gamma} \tilde{H} \frac{e^{\xi t}}{K(p)+\xi} d \xi=\int_{-i \infty}^{i \infty} \tilde{H} \frac{e^{\xi t}}{K(p)+\xi} d \xi
$$

(since $\mathfrak{R} \phi_{j}(\xi)>0$ for all $\mathfrak{R} \xi=0$, and taking into account inequality (3.18), we see that the last integral in the above formula converges absolutely). Applying the identities

$$
K^{\prime}\left(\phi_{l}\right)=-\frac{1}{\phi_{l}^{\prime}(\xi)}
$$

and using the theory of residues, we obtain

$$
\int_{-i \infty}^{i \infty} \frac{\tilde{H}\left(p, \phi_{1}, \ldots, \phi_{N}, y\right)}{K(p)+\xi} e^{p x} d p=2 \pi i \sum_{l=1}^{M} \tilde{H}\left(\phi_{l+N}, \phi_{1}, \ldots, \phi_{N}, y\right) e^{\phi_{l+N}(\xi) x} \phi_{l+N}^{\prime}(\xi)
$$

where $\phi_{l+N}$ are "negative" roots of equation for equation $K(p)=-\xi$, such that

$$
\operatorname{Re} \phi_{j}(\xi)<0, \quad \operatorname{Re} \xi>0 .
$$

For any $\phi_{k}(\xi), k=1, \ldots, N$, there exists the function $\phi_{j}(\xi), j=1, \ldots, N$, such that

$$
\phi_{k}(-\xi)=\phi_{j}(\xi), \quad \operatorname{Re} \xi=0 .
$$


Therefore taking into account symmetrical properties of function $\tilde{H}$ after the change of variable $p=\phi_{l+N}(\xi)$, we get

$$
\begin{aligned}
& \frac{1}{4 \pi^{2}} \int_{-i \infty}^{i \infty} e^{p x} \int_{-i \infty}^{i \infty} \sum_{j=1}^{N} p^{N-j} \int_{\Gamma} \frac{e^{\xi t}}{K(p)+\xi}\left(\mathbb{A}^{-1} \overrightarrow{\mathbf{B}}\right)_{j} \\
& \quad=-\frac{1}{2 \pi i} \sum_{l=1}^{M} \int_{-i \infty}^{i \infty} e^{\xi t} \tilde{H}\left(\phi_{l+N}, \phi_{1}, \phi_{2}, \ldots, \phi_{N}, y\right) e^{\phi_{l+N}(\xi) x} \phi_{l+N}^{\prime}(\xi) d \xi \\
& =-\frac{1}{2 \pi i} \sum_{l=1}^{M} \int_{\Gamma_{l}} e^{p x-K(p) t} \tilde{H}\left(p, \phi_{1}(K(p)), \phi_{2}, \ldots, \phi_{N}, y\right) d p \\
& =\frac{1}{2 \pi i} \int_{-i \infty}^{i \infty} e^{p x-K(p) t} \tilde{H}\left(p, \phi_{1}(K(p)), \phi_{2}, \ldots, \phi_{N}, y\right) d p,
\end{aligned}
$$

where

$$
\Gamma_{l}=\left\{p=\phi_{l+N}(\xi), \operatorname{Re} \xi=0\right\} .
$$

Therefore taking into account (3.15), (3.24), we obtain the following integral representation for Green function $G(x, y, t)$ of problem (3.1):

$$
\begin{gathered}
G(x, y, t)=F_{1}(x-y, t)+F_{2}(x, y, t), \\
F_{1}(x, t)=\frac{1}{2 \pi i} \int_{-i \infty}^{i \infty} e^{p x-K(p) t} d p \\
F_{2}(x, y, t)=\frac{1}{2 \pi i} \sum_{j=1}^{N} \int_{-i \infty}^{i \infty} e^{p x-K(p) t} \frac{e^{-\phi_{j}(K(p)) y} P_{N}(p)}{P_{N}^{\prime}\left(\phi_{j}\right)\left(p-\phi_{j}\right)} d p
\end{gathered}
$$

where the function $P_{N}$ is defined in (3.16).

\section{Asymptotics of the Green function}

If $K(p)=a_{n} p^{n}+a_{m} p^{m}, m>n$, then there exist $M_{1}=m / 2$ different inverse functions $\phi_{j}(\xi)=K^{-1}(-\xi)$, such that for $\xi \in D$ (see book [7]),

$$
\operatorname{Re} \phi_{j}(\xi)>0 \text {. }
$$

Moreover, the asymptotics

$$
\phi_{l}(\xi)=e^{i(\pi+2 \pi l)(1 / m)}\left(a_{m}^{-1} \xi\right)^{1 / m}+O\left(\xi^{-(1-\gamma) / m}\right)
$$

is true as $\xi \rightarrow \infty$, with $\gamma>0$. Now we consider case $\xi \rightarrow 0$.

We represent

$$
p^{n}=\frac{1}{a_{n}} \frac{-\xi}{1+O(p)} \quad \text { or } \quad p^{m-n}=\frac{a_{n}}{a_{m}}\left(-1+\frac{-\xi}{a_{n} p^{n}}+O(p)\right) \text { for }|p| \leq 1 .
$$


Hence we get the asymptotic representations for $l=1, \ldots, Q=n / 2$,

$$
\phi_{l}(\xi)=\beta_{l} \xi^{1 / n}+O\left(|\xi|^{2 / n}\right), \quad \xi \longrightarrow 0,
$$

where $\beta_{l}$ are the roots of the equation $a_{n} z^{n}+1=0$, such that $\operatorname{Re} \beta_{l} \xi^{1 / n}>0$ for all $\operatorname{Re} \xi>0$.

For $l=Q+1, \ldots, N$, we have

$$
\phi_{l+j}(\xi)=r_{j}+O\left(|\xi|^{2 / n}\right), \quad \xi \longrightarrow 0,
$$

where $r_{j}$ are the roots of the equation $a_{n}+a_{m} z^{m-n}=0$, such that $\operatorname{Re} r_{j}>0$.

We have (see (3.26))

$$
\begin{aligned}
& G(x, y, t)=\frac{1}{2 \pi i} \int_{-i \infty}^{i \infty} e^{p x-K(p) t} H(p, y) d p \\
& H(p, y)=e^{-p y}+\sum_{j=1}^{N} \frac{e^{-\phi_{j}(K(p)) y} P_{N}(p)}{P_{N}^{\prime}\left(\phi_{j}\right)\left(p-\phi_{j}\right)} .
\end{aligned}
$$

Since for $l=0, \ldots, N-1, \operatorname{Re} p=0$,

$$
\sum_{j=1}^{N} \frac{\phi_{j}^{l} P_{N}(p)}{P_{N}^{\prime}\left(\phi_{j}\right)\left(p-\phi_{j}\right)}=\frac{P_{N}(p)}{2 \pi i} \int_{-i \infty, u \neq p}^{i \infty} \frac{u^{l}}{P_{N}(u)(p-u)} d u=-p^{l}
$$

we have

$$
H(p, y)=e^{-p y}-\sum_{l=1}^{N-1} \frac{(-p y)^{l}}{l !}+\sum_{j=1}^{N} \widetilde{B}\left(\phi_{j}(K(p))\right) \frac{P_{N}(p)}{P_{N}^{\prime}\left(\phi_{j}\right)\left(p-\phi_{j}\right)},
$$

where

$$
\widetilde{B}(z)=e^{-z y}-\sum_{l=0}^{N-1}(-1)^{l} \frac{(z y)^{l}}{l !} .
$$

Therefore using (4.4)-(4.5) and symmetrical properties of function $H(p, y)$, we obtain for $|p| \ll 1$,

$$
\sum_{j=1}^{n / 2} \widetilde{B}\left(\phi_{j}(K(p))\right) \frac{P_{N}(p)}{P_{N}^{/}\left(\phi_{j}\right)\left(p-\phi_{j}\right)}=O\left(p^{Q+1} y^{Q+1}\right)
$$


Also by definition, we have

$$
\Pi_{j=1}^{N} \phi_{j}^{2}=\frac{(-1)^{N}}{a_{m}}\left(a_{n} p^{n}+a_{m} p^{m}\right), \quad a_{m} P_{N}(p) P_{N}(-p)=(-1)^{N} 2 K(p) .
$$

Therefore using (4.4)-(4.5), we get with $Q=n / 2$ that

$$
\begin{aligned}
\sum_{k=Q+1}^{N} & \widetilde{B}\left(\phi_{k}(K(p))\right) \frac{P_{N}(p)}{P_{N}^{\prime}\left(\phi_{k}\right)\left(p-\phi_{k}\right)} \\
= & \sum_{k=Q+1}^{N} \widetilde{B}\left(\phi_{k}(K(p))\right) \frac{P_{N}(p) P_{N}\left(-\phi_{k}\right)}{P_{N}^{\prime}\left(\phi_{k}\right) P_{N}\left(-\phi_{k}\right)\left(p-\phi_{k}\right)} \\
= & \sum_{k=Q+1}^{N} \widetilde{B}\left(\phi_{k}(K(p))\right) \frac{a_{m} \Pi_{j=1}^{N}\left(\phi_{j}+\phi_{k}\right)\left(\phi_{j}-p\right)}{2\left(a_{n} n \phi_{k}^{n-1}+a_{m} m \phi_{k}^{m-1}\right)\left(p-\phi_{k}\right)} \\
= & \frac{a_{m}}{2} \sum_{k=Q+1}^{N} \tilde{B}\left(\phi_{k}(K(p))\right) \frac{\Pi_{j=1}^{N} \phi_{j}^{2}+\phi_{k}^{N} \Pi_{j=1}^{N} \phi_{j}+(-p)^{N} \Pi_{j=1}^{N} \phi_{j}+\phi_{k}^{N}(-p)^{N}}{-r_{k}^{n}\left(a_{n} n+a_{m} m r_{k}^{m-n}\right)} \\
& +O\left(p^{Q+1}\left(1+y^{Q+1}\right)\right) \\
= & p^{Q} \sqrt{\frac{a_{m}}{a_{n}}} \frac{(-1)^{N / 2}}{2(m-n)} \sum_{k=1}^{N-Q} \widetilde{B}\left(r_{k}\right) r_{k}^{N-n}+O\left(p^{Q+1}\left(1+y^{Q+1}\right)\right),
\end{aligned}
$$

where $r_{k}$ was defined in (4.4), (4.5).

Substituting (4.11)-(4.13) into (4.7), we easily get

$$
H(p, y)=D_{1} p^{n / 2} B(y)+O\left(p^{n / 2+1}\left(1+y^{n / 2+1}\right)\right),
$$

where constant $D_{1}$ is defined by

$$
D_{1}=\sqrt{\frac{i^{m} a_{m}}{i^{n} a_{n}}} \frac{i^{n / 2}}{2(m-n)},
$$

and function

$$
B(y)=\sum_{j=1}^{N-n / 2} r_{j}^{N-n}\left(e^{-r_{j} y}-\sum_{l=0}^{n / 2}(-1)^{l} \frac{\left(r_{j} y\right)^{l}}{l !}\right) .
$$


Note that for $|p|>1$,

$$
|H(p, y)|<C \text {. }
$$

Making the change of variable $p^{n} t=z^{n}$, we obtain for Green function with $x_{1}=x t^{-1 / n}$ that

$$
\begin{aligned}
G(x, y, t)= & \frac{1}{2 \pi i} \int_{-i \infty}^{i \infty} e^{p x-K(p) t} H(p, y) d p \\
= & \frac{1}{2 \pi i}\left(\int_{-i}^{i} e^{p x-a_{n} p^{n} t}\left(D_{1} p^{Q} B(y)+O\left(p^{Q+1}\left(1+y^{Q+1}\right)\right)\right) d p\right. \\
& +\int_{-i}^{i} e^{p x-a_{n} p^{n} t}\left(e^{-C p^{m} t}-1\right) O\left(p^{Q}\left(1+y^{Q+1}\right)\right) d p \\
& \left.+\int_{-i \infty,|p|>1}^{i \infty} e^{p x-K(p) t} H(p, y) d p\right) \\
= & \mathscr{F}(x, y, t)+R(x, y, t),
\end{aligned}
$$

where

$$
\begin{aligned}
& \mathscr{F}(x, y, t)=\frac{1}{2 \pi i} D_{1} t^{-(Q+1) / n} B(y) \int_{-i \infty}^{i \infty} e^{z x_{1}-a_{n} z^{n}} z^{Q} d z \\
&= B(y) t^{-(Q+1) / n} \Lambda\left(x_{1}\right) \\
& R(x, y, t)=\frac{1}{2 \pi i}\left(\int_{-i}^{i} e^{p x-a_{n} p^{n} t} O\left(p^{Q+1}\left(1+y^{Q+1}\right)\right) d p\right. \\
&+\int_{-i}^{i} e^{p x-a_{n} p^{n} t}\left(e^{-C p^{m} t}-1\right) O\left(p^{Q}\left(1+y^{Q+1}\right)\right) d p \\
&\left.+\int_{-i \infty,|p|>1}^{i \infty} e^{p x-K(p) t} H(p, y) d p\right)=O\left(\langle y\rangle^{Q+1} t^{-(Q+2) / n}\right) .
\end{aligned}
$$

We also note that for $t>0, w \geq 0, k=0,1, r \geq 1, Q=n / 2$,

$$
\left\|(\cdot) t^{-(Q+1) / n} \partial_{x}^{k} \Lambda\left((\cdot) t^{-1 / n}\right)\right\|_{\mathbf{L}^{r}} \leq C t^{-(Q+k-w) / n+(1 / n)(1 / r-1)} .
$$

In the same way, we can obtain asymptotic formula for the function $G_{1}$, which was defined in the introduction,

$$
\begin{gathered}
G_{1}(s, q)=t^{-(Q+1) / n} y^{Q} \Lambda\left(x_{1}\right) O\left(\langle y\rangle^{Q+1} t^{-(Q+2) / n}\right), \\
A_{j}=\prod_{k=1, k \neq j}^{n / 2} \frac{1+r_{k}}{1-r_{k}}, \quad a_{n} r_{k}^{n}=-1, \operatorname{Re} r_{k}>0 .
\end{gathered}
$$




\section{Preliminaries}

We introduce the operators

$$
\begin{gathered}
\mathbb{G}(t) f=\int_{0}^{+\infty} G(x, y, t) f(y) d y, \\
G(x, y, t)=F_{1}(x-y, t)+F_{2}(x, y, t), \\
F_{1}(x, t)=\frac{1}{2 \pi i} \int_{-i \infty}^{i \infty} e^{p x-K(p) t} d p \\
F_{2}(x, y, t)=\frac{1}{2 \pi i} \sum_{j=1}^{N} \int_{-i \infty}^{i \infty} e^{p x-K(p) t} \frac{e^{-\phi_{j}(K(p)) y} P_{N}(p)}{P_{N}^{\prime}\left(\phi_{j}\right)\left(p-\phi_{j}\right)} d p \\
P_{N}(p)=\prod_{l=1}^{N}\left(p-\phi_{l}\right) .
\end{gathered}
$$

Also we denote

$$
\begin{aligned}
& G_{0}(s)=\frac{1}{2 \pi i} i^{n / 2} \int_{-i \infty}^{i \infty} e^{z s-a_{n} z^{n}} z^{n / 2} d z, \quad D=\sqrt{\frac{i^{m} a_{m}}{i^{n} a_{n}}} \frac{1}{2(m-n)}>0, \\
& \mathscr{G}_{0}(t) \phi=t^{-1 / n} \int_{\mathbb{R}^{n}} G_{1}\left(x t^{-1 / n}, y t^{-1 / n}\right) \phi(y) d y, \\
& G_{1}(s, q)=\frac{1}{2 \pi i} \int_{-i \infty}^{i \infty} e^{-a_{n} z^{n}}\left(e^{z(s-q)}-\sum_{j=1}^{n / 2} A_{j} e^{-\left(s+r_{j} q\right)}\right) d z, \\
& A_{j}=\prod_{k=1, k \neq j}^{n / 2} \frac{1+r_{k}}{1-r_{k}}, \quad a_{n} r_{k}^{n}=-1, \operatorname{Re} r_{k}>0, \\
& B(x)=\sum_{j=1}^{N-(n / 2)} r_{j}^{N-n}\left(e^{-r_{j} x}-\sum_{l=0}^{n / 2}(-1)^{l} \frac{\left(r_{j} x\right)^{l}}{l !}\right) .
\end{aligned}
$$

We first collect some preliminary estimates of the Green operator $\mathbb{G}(t)$ in the norms $\|\phi\|_{\mathbf{L}^{r}}$ and $\|\phi\|_{\mathrm{L}^{1, a},}$, where $a \in(0, Q+1], r=1, \infty$. Everywhere below, the constants $C>0$ depend only on the characteristics of the symbol $K(p)$, that is, on the numbers $n, m, a_{n}$, and $a_{m}$.

Lemma 5.1. Suppose that the function $\phi \in \mathbf{L}^{\infty} \cap \mathbf{L}^{1, Q+1}$. Then the estimates

$$
\begin{aligned}
& \left\|\partial_{x}^{k} \mathbb{G}(t) \phi\right\|_{\mathbf{L}^{r}} \leq C\{t\}^{-k / m}\langle t\rangle^{-k / n-1 / n(1-1 / r)}\left(\|\phi\|_{\mathbf{L}^{\infty}}+\|\phi\|_{\mathbf{L}^{1}}+\langle t\rangle^{-1 / n}\|\phi\|_{\mathbf{L}^{1,1}}\right), \\
& \left\|(\cdot)^{\omega} \partial_{x}^{k}\left(\mathbb{G}(t) \phi-\theta t^{-(Q+1) / n} G_{0}\left(t^{-1 / n}(\cdot)\right)\right)\right\|_{\mathbf{L}^{r}} \\
& \quad \leq C\langle t\rangle^{-(Q+1+k-w) / n+(1 / n)(1 / r-1)}\{t\}^{-(k-w) / m+(1 / m)(1 / r-1)}\|\phi\|_{\mathbf{L}^{1, Q+1}}
\end{aligned}
$$


are valid for all $t>0$, where

$$
1 \leq r \leq \infty, \quad 0 \leq \omega \leq Q+1, \theta=\int_{0}^{+\infty} x^{Q} \phi(x) d x, \quad k=0,1, Q=\frac{n}{2} .
$$

Proof. By symbol $\overline{\mathscr{F}}_{p \rightarrow x}$, we denote inverse Fourier transformation. Note that the kernel $F_{1}(x, t)=\overline{\mathscr{F}}_{p \rightarrow x}\left(e^{-K(p) t}\right)$ in representation (5.3) is a smooth function $F_{1}(x, t) \in \mathbf{C}^{\infty}\left(\mathbb{R}^{1}\right)$ and decays at infinity so that

$$
\sup _{x \in \mathbb{R}^{1}}\left\langle x\langle t\rangle^{-1 / n}\{t\}^{-1 / m}\right\rangle^{2+k}\left|\partial_{x}^{k} F_{1}(x, t)\right| \leq C\langle t\rangle^{-(1+k) / n}\{t\}^{-(1+k) / m},
$$

for all $k=0,1,2$. Indeed, we have

$$
\begin{aligned}
& \left|\partial_{x}^{k} F_{1}(x, t)\right|=\left|\overline{\mathscr{F}}_{p \rightarrow x}\left(p^{k} e^{-K(p) t}\right)\right| \leq\left. C|| p^{k} e^{-K(p) t}\right|_{\mathbf{L}_{p}^{1}} \leq\langle t\rangle^{-(1+k) / n}\{t\}^{-(1+k) / m}, \\
& \left|x^{2+k} \partial_{x}^{k} F_{1}(x, t)\right|=\left|\overline{\mathscr{F}}_{p \rightarrow x}\left(\partial_{p}^{2+k}\left(p^{k} e^{-K(p) t}\right)\right)\right| \leq C|| \partial_{p}^{2+k}\left(p^{k} e^{-K(p) t}\right) \|_{\mathbf{L}_{p}^{1}}
\end{aligned}
$$

for $k=0,1,2$. Since

$$
\left|\partial_{p}^{2+k}\left(p^{k} e^{-K(p) t}\right)\right| \leq C t\{p\}^{n-2}\langle p\rangle^{m-2} e^{-R e} K(p) t
$$

for all $p \in \mathbb{R}, k=0,1,2$, we obtain

$$
\left|x^{2+k} \partial_{x}^{k} F_{1}(x, t)\right| \leq C\langle t\rangle^{1 / n}\{t\}^{1 / m}
$$

Therefore estimate (5.9) is true. By virtue of (5.9), we find

$$
\left\|\partial_{x}^{k} F_{1}(\cdot, t)\right\|_{\mathbf{L}^{1}} \leq C\langle t\rangle^{-(1+k) / n}\{t\}^{-(1+k) / m}\left\|\left\langle x\langle t\rangle^{-1 / n}\{t\}^{-1 / m}\right\rangle^{2+k}\right\|_{\mathbf{L}_{x}^{1}} \leq C\langle t\rangle^{-k / n}\{t\}^{-k / m} .
$$

Therefore using the Young inequality from (5.9) and (5.13), we find

$$
\left\|\int_{0}^{+\infty} \partial_{x}^{k} F_{1}(\cdot-y, t) \phi(y) d y\right\|_{\mathbf{L}^{r}} \leq C\{t\}^{-k / m}\langle t\rangle^{-k / n-1 / n(1-1 / r)}\left(\|\phi\|_{\mathbf{L}^{\infty}}+\|\phi\|_{\mathbf{L}^{1}}\right) .
$$

From (4.2), (4.4), and (4.5), we have

$$
\begin{aligned}
& \left|\sum_{j=1}^{N} \frac{e^{-\phi_{j}(K(p)) y} P_{N}(p)}{P_{N}^{\prime}\left(\phi_{j}(K(p))\right)\left(p-\phi_{j}\right)}\right|<C, \quad|p| \leq 1, \\
& \left|\sum_{j=1}^{N} \frac{e^{-\phi_{j}(K(p)) y} P_{N}(p)}{P_{N}^{\prime}\left(\phi_{j}(K(p))\right)\left(p-\phi_{j}\right)}\right|<C e^{-C p y}, \quad|p|>1 .
\end{aligned}
$$


Therefore, we obtain

$$
\begin{aligned}
&\left\|\int_{0}^{+\infty} \partial_{x}^{k} F_{2}(\cdot, y, t) \phi(y) d y\right\|_{\mathbf{L}^{\infty}} \\
& \quad \leq C\|\phi\|_{\mathbf{L}^{1}} \int_{-i}^{i} e^{-\operatorname{Re} K(p) t}|p|^{k}|d p| \\
&+\int_{|p|>1, p \in \mathscr{C}_{1}} e^{-\operatorname{Re} K(p) t}|p|^{k}|d p| \int_{0}^{C y} d y e^{\operatorname{Re} p(x-C y)-\operatorname{Re} K(p) t}|\phi(y)| \\
&+\int_{|p|>1, p \in \mathscr{C}_{2}} e^{-\operatorname{Re} K(p) t}|p|^{k}|d p| \int_{C y}^{+\infty} d y e^{\operatorname{Re} p(x-C y)-\operatorname{Re} K(p) t}|\phi(y)|,
\end{aligned}
$$

where

$$
\begin{aligned}
& \mathscr{C}_{1}=\left\{p=\rho e^{ \pm i \lambda_{1}} \mid \rho \geq 0, \lambda_{1}=\frac{\pi}{2}+\varepsilon, \operatorname{Re} K(p)>0, \varepsilon>0\right\}, \\
& \mathscr{C}_{2}=\left\{p=\rho e^{ \pm i \lambda_{1}} \mid \rho \geq 0, \lambda_{1}=\frac{\pi}{2}-\varepsilon, \operatorname{Re} K(p)>0, \varepsilon>0\right\} .
\end{aligned}
$$

We make the change of variable $|p|^{n} t=z$ to get

$$
\int_{-i}^{i} e^{-\operatorname{Re} K(p) t}|p|^{k}|d p| \leq C t^{-(k+1) / n} .
$$

Also taking $|p|^{m} t=z$, we find

$$
\begin{aligned}
& \int_{|p|>1, p \in \mathscr{C}_{1}} e^{-\Re K(p) t}|p|^{k}|d p| \int_{0}^{C y} d y e^{\operatorname{Re} p(x-C y)}|\phi(y)| \\
& \quad+\int_{|p|>1, p \in \mathscr{C}_{2}} e^{-\operatorname{Re} K(p) t}|p|^{k}|d p| \int_{C y}^{+\infty} d y e^{\operatorname{Re} p(x-C y)-\operatorname{Re} K(p) t}|\phi(y)| \\
& \leq C t^{-(k+1) / m} \int_{0}^{+\infty} e^{-C_{1} t^{-1 / m}|x-C y|}|\phi(y)||d y| \leq C t^{-k / m}\|\phi\|_{\mathbf{L}^{1}} .
\end{aligned}
$$

Via (5.16), the estimates (5.18) and (5.19) imply that

$$
\left\|\int_{0}^{+\infty} \partial_{x}^{k} F_{2}(\cdot, y, t) \phi(y) d y\right\|_{\mathbf{L}^{\infty}} \leq C\{t\}^{-k / m}\langle t\rangle^{-(k+1) / n}\|\phi\|_{\mathbf{L}^{1}} .
$$

Since

$$
\frac{1}{2 \pi i} \sum_{j=1}^{N} \int_{-i \infty}^{i \infty} e^{p x-K(p) t} \frac{P_{N}(p)}{P_{N}^{\prime}\left(\phi_{j}\right)\left(p-\phi_{j}\right)} d p=\frac{1}{2 \pi i} \int_{-i \infty}^{-i \infty} e^{p x-K(p) t} d p=F_{1}(x, 0, t),
$$

we rewrite function $F_{2}(x, y, t)$ in following form:

$$
F_{2}=F_{1}(x, 0, t)+F_{3}(x, y, t) .
$$


By virtue of (4.2)-(4.5), we have

$$
\left|\sum_{j=1}^{N} \frac{\left(e^{-\phi_{j}(K(p)) y}-1\right) P_{N}(p)}{P_{N}^{\prime}\left(\phi_{j}(K(p))\right)\left(p-\phi_{j}\right)}\right|<C\{p\} y+C .
$$

So changing the contour of integration, we get

$$
\begin{aligned}
\left\|\partial_{x}^{k} F_{3}(\cdot, y, t)\right\|_{\mathbf{L}_{x}^{1}} & \leq C\left(y \int_{|p|<1, p \in \mathscr{C}_{1}}|p|^{k} e^{-\operatorname{Re} K(p) t} d p+\int_{|p|>1, p \in \mathscr{C}_{1}}|p|^{k-1} e^{-\operatorname{Re} K(p) t}|d p|\right) \\
& \leq\{t\}^{-k / m}\langle t\rangle^{-(k+1) / n} y,
\end{aligned}
$$

where

$$
\mathscr{C}_{1}=\left\{p=\rho e^{ \pm i \lambda_{1}} \mid \rho \geq 0, \lambda_{1}=\frac{\pi}{2}+\varepsilon, \operatorname{Re} K(p)>0, \varepsilon>0\right\} .
$$

Therefore from (5.13) and (5.24), we get

$$
\left\|\partial_{x}^{k} F_{2}(\cdot, y, t)\right\|_{\mathbf{L}_{x}^{1}}<C\{t\}^{-k / m}\langle t\rangle^{-(k+1) / n} y+\{t\}^{-k / m}\langle t\rangle^{-k / n}
$$

Hence, it follows that

$$
\left\|\int_{0}^{+\infty} \partial_{x}^{k} F_{2}(\cdot, y, t) \phi(y) d y\right\|_{\mathbf{L}^{1}} \leq C\{t\}^{-k / m}\langle t\rangle^{-k / n}\left(\|\phi\|_{\mathbf{L}^{1}}+\langle t\rangle^{-k / n}\|\phi\|_{\mathbf{L}^{1,1}}\right) .
$$

From (5.14), (5.20), (5.27), we obtain the first estimate of the lemma:

$$
\left\|\partial_{x}^{k} \mathbb{G}(t) \phi\right\|_{\mathbf{L}^{r}} \leq C\{t\}^{-k / m}\langle t\rangle^{-k / n-(1 / n)(1-1 / r)}\left(\|\phi\|_{\mathbf{L}^{\infty}}+\|\phi\|_{\mathbf{L}^{1}}+\langle t\rangle^{-1 / n}\|\phi\|_{\mathbf{L}^{1,1}}\right)
$$

for all $t>0$, where $1 \leq r \leq \infty$. Since by virtue of the estimate (4.18),

$$
\begin{aligned}
& G(x, y, t)-t^{-(Q+1) / n} G_{0}\left(t^{-1 / n}(\cdot)\right) B(y) \\
& =\frac{1}{2 \pi i}\left(\int_{p \in \mathscr{C},|p|<1} e^{p x-a_{n} p^{n} t} O\left(p^{Q+1}\left(1+y^{Q+1}\right)\right) d p\right. \\
& \quad+\int_{p \in \mathscr{C},|p|<1} e^{p x-a_{n} p^{n} t}\left(e^{-C p^{m} t}-1\right) O\left(p^{Q}\left(1+y^{Q+1}\right)\right) d p \\
& \left.\quad+\int_{p \in \mathscr{C},|p| \geq 1} e^{p x-K(p) t} H(p, y) d p\right), \\
& H(p, y)<C, \quad|p|>1, p \in \mathscr{C},
\end{aligned}
$$


we easily obtain

$$
\begin{aligned}
&\left\|(\cdot)^{\omega} \partial_{x}^{k}\left(\mathbb{G}(t) \phi-9 t^{-(Q+1) / n} G_{0}\left(t^{-1 / n}(\cdot)\right)\right)\right\|_{\mathbf{L}^{r}} \\
& \leq\left\langle y^{Q+1}\right\rangle\left\|(\cdot)^{\omega} \int_{p \in \mathscr{C},|p|<1} e^{\operatorname{Re} p x-\operatorname{Re} a_{n} p^{n} t}|p|^{Q+1+k}|d p|\right\|_{\mathbf{L}^{r}} \\
& \quad+\left\|(\cdot)^{\omega} \int_{p \in \mathscr{C},|p| \geq 1} e^{\operatorname{Re} p x-K(p) t}|p|^{k}|d p|\right\|_{\mathbf{L}^{r}} \\
& \leq\left\langle y^{Q+1}\right\rangle \int_{p \in \mathscr{C},|p|<1} e^{-\operatorname{Re} a_{n} p^{n} t}|p|^{Q+1+k-w-1 / r}|d p| \\
& \quad+\int_{p \in \mathscr{C},|p| \geq 1} e^{-K(p) t}|p|^{k-w-1 / r}|d p| \\
& \leq C\langle t\rangle^{-(Q+1+k-w) / n+1 / n(1 / r-1)}\{t\}^{-(k-w) / m+1 / m(1 / r-1)}\|\phi\|_{\mathbf{L}^{1, Q+1}}
\end{aligned}
$$

for any $\omega \in[0, Q+1]$. Thus the second estimate of the lemma follows. Lemma 5.1 is proved.

In the same way, we can prove the following.

Lemma 5.2. Suppose that the function $\phi \in \mathbf{L}^{\infty} \cap \mathbf{L}^{1, Q+1}$. Then the estimates

$$
\begin{aligned}
& \left\|\partial_{x}^{k c} \varphi_{0}(t) \phi\right\|_{\mathbf{L}^{r}} \leq C\{t\}^{-k / m}\langle t\rangle^{-k / n-(1 / n)(1-1 / r)}\left(\|\phi\|_{\mathbf{L}^{\infty}}+\|\phi\|_{\mathbf{L}^{1}}+\langle t\rangle^{-1 / n}\|\phi\|_{\mathbf{L}^{1,1}}\right), \\
& \left\|(\cdot)^{\omega} \partial_{x}^{k}\left(\varphi_{0}(t) \phi-\theta t^{-(Q+1) / n} G_{0}\left(t^{-1 / n}(\cdot)\right)\right)\right\|_{\mathbf{L}^{r}} \\
& \quad \leq C\langle t\rangle^{-(Q+1+k-w) / n+(1 / n)(1 / r-1)}\{t\}^{-(k-w / m)+(1 / m)((1 / r)-1)}\|\phi\|_{\mathbf{L}^{1, Q+1}}
\end{aligned}
$$

are valid for all $t>0$, where

$$
1 \leq r \leq \infty, \quad 0 \leq \omega \leq Q+1, \theta=\int_{0}^{+\infty} x^{Q} \phi(x) d x, \quad k=0,1 .
$$

We introduce the function space for $k=0,1$,

$$
\begin{aligned}
& \|\phi\|_{\mathbf{X}}=\sup _{t>0}\left(\sum_{k=0}^{1}\left(\{t\}^{k / m}\langle t\rangle^{(Q+k) / n+(1 / n)(1-1 / r)}\left\|\partial_{x}^{k} \phi(t)\right\|_{\mathbf{L}^{r}}+\{t\}^{k / m}\langle t\rangle^{(k-1) / n}\left\|\partial_{x}^{k} \phi(t)\right\|_{\mathbf{L}^{1, Q+1}}\right)\right), \\
& \|\phi\|_{\mathbf{Y}}=\sup _{t>0}\left(\{t\}^{\sigma / m}\langle t\rangle^{Q / n+1+(1 / n)(1-1 / r)}\|\phi(t)\|_{\mathbf{L}^{r}}+\{t\}^{\sigma / m}\langle t\rangle^{1-1 / n}\|\phi(t)\|_{\mathbf{L}^{1, Q+1}}\right),
\end{aligned}
$$

where $\sigma \leq m$. 
Lemma 5.3. Let the function $f(x, t)$ satisfy $\int_{0}^{+\infty} x^{Q} f(x, t) d x=0$. Then the inequality

$$
\begin{gathered}
\left\|\int_{0}^{t} \varphi_{0}(t-\tau) f(\tau) d \tau\right\|_{\mathbf{X}} \leq C\|f\|_{\mathrm{Y}}, \\
\left\|\langle t\rangle^{\gamma_{2}} \int_{0}^{t}\left(\varphi(t-\tau)-\varphi_{0}(t-\tau)\right) f(\tau) d \tau\right\|_{\mathbf{X}} \leq C\|f(t)\|_{\mathbf{Y}}, \quad \gamma_{2} \in\left[0,1-\frac{\sigma}{m}\right),
\end{gathered}
$$

is valid for $l=0,1$, provided that the right-hand side is finite.

Proof. By Lemma 5.2, we get

$$
\begin{aligned}
& \left\|\int_{0}^{t} \partial_{x}^{k} \varphi_{0}(t-\tau) f(\tau) d \tau\right\|_{\mathbf{L}^{\infty}}+\| \int_{0}^{t} \partial_{x}^{k c G_{0}(t-\tau) f(\tau) d \tau \|_{\mathbf{L}^{1, Q+1}}} \\
& \quad \leq C\|f(t)\|_{\mathbf{Y}} \int_{0}^{1}(1+\tau)^{-1}\{\tau\}^{-\sigma / m}\{t-\tau\}^{-1 / m} d \tau \leq C\|f(t)\|_{\mathbf{Y}}
\end{aligned}
$$

for all $0 \leq t \leq 1$. We now consider $t>1$. By virtue of Lemma 5.2 with $\omega=0$, we obtain

$$
\begin{aligned}
& \left\|\int_{0}^{t} \partial_{x}^{k c} \varphi_{0}(t-\tau) f(\tau) d \tau\right\|_{\mathbf{L}^{r}} \\
& \leq C \int_{0}^{t / 2}(t-\tau)^{-(Q+1+k) / n+(1 / n)(1 / r-1)}\langle\tau\rangle^{-1+1 / n}\{\tau\}^{-\sigma / m} d \tau\|f(\tau)\|_{\mathbf{L}^{1, Q+1}} \\
& \quad+C \int_{t / 2}^{t}(t-\tau)^{-k / n-(1 / n)(1-1 / r)}\langle t\rangle^{-Q / n-1} d \tau \sup _{\tau>0}\left(\|f(\tau)\|_{\mathbf{L}^{\infty}}+\|f(\tau)\|_{\mathbf{L}^{1}}\right) \\
& \quad+C \int_{t / 2}^{t}(t-\tau)^{-(k+1) / n-(1 / n)(1-1 / r)}\langle t\rangle^{-Q / n-1+1 / n(Q+1)} d \tau \sup _{\tau>0}\|f(\tau)\|_{\mathbf{L}^{1,1}} \\
& \leq C\{t\}^{-k / m}\langle t\rangle^{-(Q+k) / n+(1 / n)(1 / r-1)}\|f\|_{\mathbf{Y}}
\end{aligned}
$$

for $1 \leq r \leq \infty$, and using the second estimate of Lemma 5.2 with $\omega=Q+1$, we get

$$
\begin{aligned}
& \left\|\int_{0}^{t} \partial_{x}^{k} \varphi_{0}(t-\tau) f(\tau) d \tau\right\|_{\mathbf{L}^{1, Q+1}} \\
& \quad \leq C \int_{0}^{t / 2}\{\tau\}^{-\sigma / m}\langle\tau\rangle^{-1+1 / n}(t-\tau)^{(1 / n)(1 / r-1-k)} d \tau\|f(\tau)\|_{\mathbf{L}^{1, Q+1}} \\
& \quad+C \int_{t / 2}^{t}\langle\tau\rangle^{-1+1 / n}(t-\tau)^{(1 / n)(1 / r-1-k)} d \tau\|f(\tau)\|_{\mathbf{L}^{1, Q+1}} \\
& \leq C\{t\}^{-k / m}\langle t\rangle^{(1-k) / n}\|f\|_{\mathbf{Y}}
\end{aligned}
$$

for all $t>1$. Hence the result of the lemma follows. In the same way, we can prove second estimate of this lemma. Lemma 5.3 is proved. 


\section{Proof of Theorem 1.1}

The local existence of solutions for the initial-boundary value problem (1.1) can be obtained by the standard contraction mapping principle (for the proof see [7]).

Theorem 6.1. Let the initial data $u_{0} \in \mathbf{L}^{\infty} \cap \mathbf{L}^{1, n / 2+1}$. Then for some $T>0$, there exists a unique solution $u(t, x) \in \mathbf{C}\left([0, T) ; \mathbf{L}^{\infty} \cap \mathbf{L}^{1, n / 2+1}\right) \cap \mathbf{C}\left((0, T) ; \mathbf{W}_{\infty}^{1,0} \cap \mathbf{W}_{1}^{1, n / 2+1}\right)$ of the problem (1.1).

By the local existence theorem (Theorem 6.1), it follows that the global solution (if it exists) is unique. So our main purpose in the proof of Theorem 1.1 is to show the globalin-time existence of solutions.

We use Theorem 2.1 to prove Theorem 1.1. Denote

$$
\|g\|_{\mathbf{Z}}=\left(\|g(t)\|_{\mathbf{L}^{\infty}}+\|g(t)\|_{\mathbf{L}^{1,1+n / 2}}\right)
$$

and

$$
\begin{array}{r}
\|\phi\|_{\mathbf{X}}=\sup _{t>0}\left(\sum _ { k = 0 } ^ { 1 } \left(\{t\}^{k / m}\langle t\rangle^{(Q+k) / n+(1 / n)(1-1 / r)}\left\|\partial_{x}^{k} \phi(t)\right\|_{\mathbf{L}^{r}}\right.\right. \\
\left.\left.+\{t\}^{k / m}\langle t\rangle^{(k-1) / n}\left\|\partial_{x}^{k} \phi(t)\right\|_{\mathbf{L}^{1, Q+1}}\right)\right),
\end{array}
$$

and

$$
\|\phi\|_{\mathbf{Y}}=\sup _{t>0}\left(\{t\}^{\sigma / m}\langle t\rangle^{Q / n+1+(1 / n)(1-1 / r)}\|\phi(t)\|_{\mathbf{L}^{r}}+\{t\}^{\sigma / m}\langle t\rangle^{1-1 / n}\|\phi(t)\|_{\mathbf{L}^{1, Q+1}}\right) .
$$

From Lemmas 5.1 and 5.2, we easily get the following estimates:

$$
\left\|\langle t\rangle^{\gamma}\left(\mathscr{G}(t)-\mathscr{G}_{0}(t)\right) \phi\right\|_{\mathbf{X}} \leq C\|\phi\|_{\mathrm{Z}}
$$

where $\gamma=\min ((1 / 2) \mu, 1-\sigma / m)$. From Lemma 5.3, we have that if $\int_{\mathbb{R}^{+}} x^{Q} f d x=0$,

$$
\begin{gathered}
\left\|\int_{0}^{t} \mathscr{G}_{0}(t-\tau) f(\tau) d \tau\right\|_{\mathbf{X}} \leq C\|f(t)\|_{\mathbf{Y}}, \quad \nu \in\left[0,1-\frac{\sigma}{m}\right], \\
\left\|\langle t\rangle^{\gamma} \int_{0}^{t}\left(\mathscr{G}(t-\tau)-\mathscr{G}_{0}(t-\tau)\right) f(\tau) d \tau\right\|_{\mathbf{X}} \leq C\|f(t)\|_{\mathbf{Y}} .
\end{gathered}
$$

Also by direct calculation, we have

$$
\begin{gathered}
\left\|\left(\mathcal{N}\left(u_{1}\right)-\mathcal{N}\left(u_{2}\right)\right)\right\|_{\mathbf{Y}} \leq C\left\|\left(u_{1}-u_{2}\right)\right\|_{\mathbf{X}}\left(\left\|u_{1}\right\|_{\mathbf{X}}^{\sigma+\rho-1}+\left\|u_{2}\right\|_{\mathbf{X}}^{\sigma+\rho-1}\right), \\
\left\|t^{-Q+1 / n} V\left((\cdot) t^{-1 / n}\right)\right\|_{\mathbf{X}} \leq C\|V(\cdot)\|_{\mathbf{W}},
\end{gathered}
$$


where

$$
\|\phi\|_{\mathrm{W}}=\sup _{t>0}\left(\|\phi(t)\|_{\mathbf{L}^{r}}+\|\phi(t)\|_{\mathbf{L}^{1, Q+1}}\right) .
$$

Therefore from Theorem 2.1 with $\beta=1 / n, \alpha=(n+2) / 2 n$, and

$$
G_{0}(s)=\frac{1}{2 \pi i} i^{Q} \int_{-i \infty}^{i \infty} e^{z s-a_{n} z^{n}} z^{Q} d z, \quad D=\sqrt{\frac{i^{m} a_{m}}{i^{n} a_{n}}} \frac{1}{2(m-n)}>0,
$$

we get the result of Theorem 1.1. This completes the proof of Theorem 1.1.

\section{Acknowledgments}

This work is partially supported by CONACYT. We are grateful to an unknown referee for many useful suggestions and comments.

\section{References}

[1] L. Debnath, Nonlinear Partial Differential Equations for Scientists and Engineers, 2nd ed., Birkhäuser Boston, Massachusetts, 2005.

[2] M. Escobedo and O. Kavian, Asymptotic behaviour of positive solutions of a nonlinear heat equation, Houston Journal of Mathematics 14 (1988), no. 1, 39-50.

[3] M. Escobedo, O. Kavian, and H. Matano, Large time behavior of solutions of a dissipative semilinear heat equation, Communications in Partial Differential Equations 20 (1995), no. 7-8, 14271452.

[4] V. A. Galaktionov, S. P. Kurdyumov, and A. A. Samarskii, Asymptotic eigenfunctions of the Cauchy problem for a nonlinear parabolic equation, Mathematics of the USSR-Sbornik 54 (1986), 421455.

[5] V. A. Galaktionov and J. L. Vázquez, Asymptotic behaviour of nonlinear parabolic equations with critical exponents. A dynamical systems approach, Journal of Functional Analysis 100 (1991), no. $2,435-462$.

[6] A. Gmira and L. Véron, Large time behaviour of the solutions of a semilinear parabolic equation in $\mathbf{R}^{N}$, Journal of Differential Equations 53 (1984), no. 2, 258-276.

[7] N. Hayashi and E. I. Kaikina, Nonlinear Theory of Pseudodifferential Equations on a Half-Line, North-Holland Mathematics Studies, vol. 194, Elsevier Science B.V., Amsterdam, 2004.

[8] N. Hayashi, E. I. Kaikina, and P. I. Naumkin, Large-time behaviour of solutions to the dissipative nonlinear Schrödinger equation, Proceedings of the Royal Society of Edinburgh. Section A. Mathematics 130 (2000), no. 5, 1029-1043.

[9] __ Global existence and time decay of small solutions to the Landau-Ginzburg type equations, Journal d'Analyse Mathématique 90 (2003), 141-173.

[10] N. Hayashi, E. I. Kaikina, and H. F. Ruiz Paredes, Boundary-value problem for the Kortewegde Vries-Burgers type equation, NoDEA. Nonlinear Differential Equations and Applications 8 (2001), no. 4, 439-463.

[11] N. Hayashi, E. I. Kaikina, and I. A. Shishmarev, Asymptotics of solutions to the boundary-value problem for the Korteweg-de Vries-Burgers equation on a half-line, Journal of Mathematical Analysis and Applications 265 (2002), no. 2, 343-370.

[12] S. Kamin and L. A. Peletier, Large time behaviour of solutions of the porous media equation with absorption, Israel Journal of Mathematics 55 (1986), no. 2, 129-146.

[13] O. Kavian, Remarks on the large time behaviour of a nonlinear diffusion equation, Annales de l'Institut Henri Poincaré. Analyse Non Linéaire 4 (1987), no. 5, 423-452. 


\section{Critical dissipative equations}

[14] P. I. Naumkin and I. A. Shishmarëv, Nonlinear Nonlocal Equations in the Theory of Waves, Translations of Mathematical Monographs, vol. 133, American Mathematical Society, Rhode Island, 1994.

[15] E. Zuazua, Some recent results on the large time behavior for scalar parabolic conservation laws, Elliptic and Parabolic Problems. Proceedings of the 2nd European conference, (Pont-à-Mousson, 1994), Pitman Res. Notes Math. Ser., vol. 325, Longman Sci. Tech., Harlow, 1995, pp. 251-263.

Elena I. Kaikina: Instituto de Matemáticas Unidad Morelia, Universidad Nacional Autónoma de México (UNAM), Campus Morelia, AP 61-3 (Xangari), CP 58180, Morelia Michoacán, Mexico E-mail address: ekaikina@matmor.unam.mx 


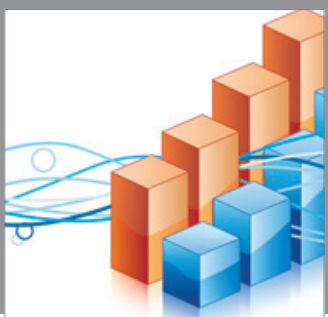

Advances in

Operations Research

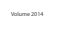

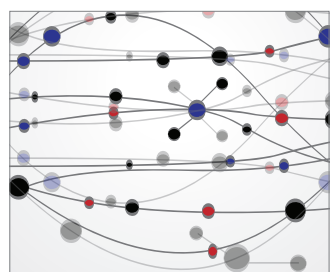

\section{The Scientific} World Journal
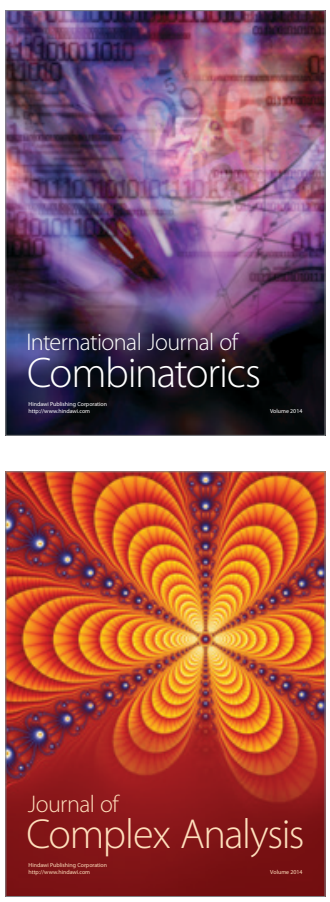

International Journal of

Mathematics and

Mathematical

Sciences
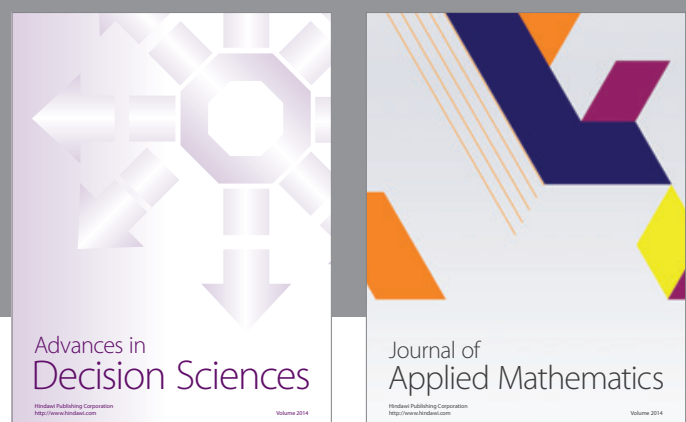

Journal of

Applied Mathematics
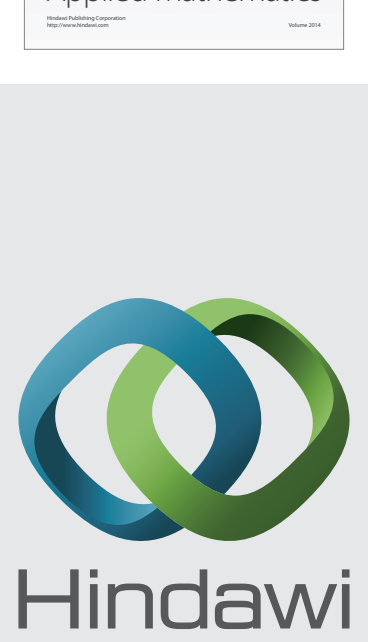

Submit your manuscripts at http://www.hindawi.com
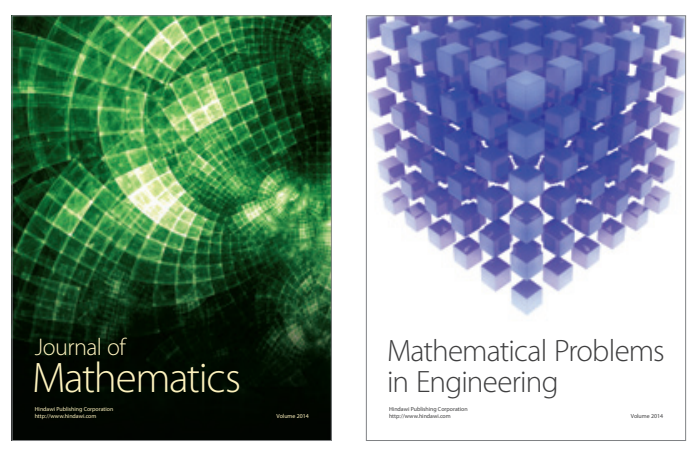

Mathematical Problems in Engineering
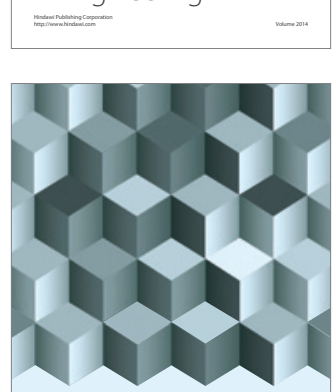

Journal of

Function Spaces
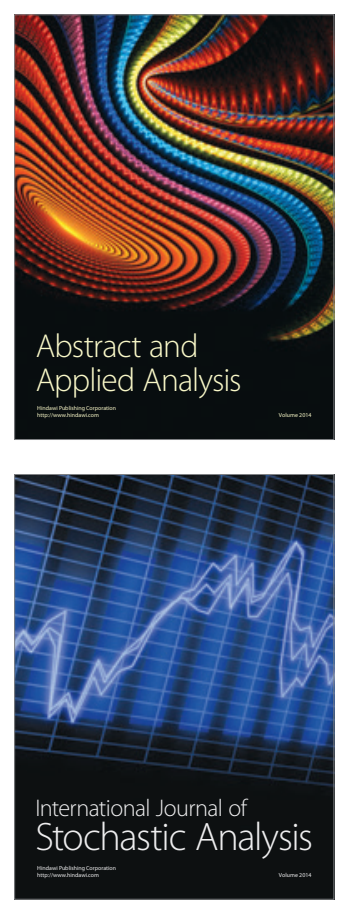

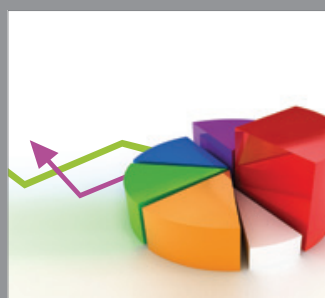

ournal of

Probability and Statistics

Promensencen
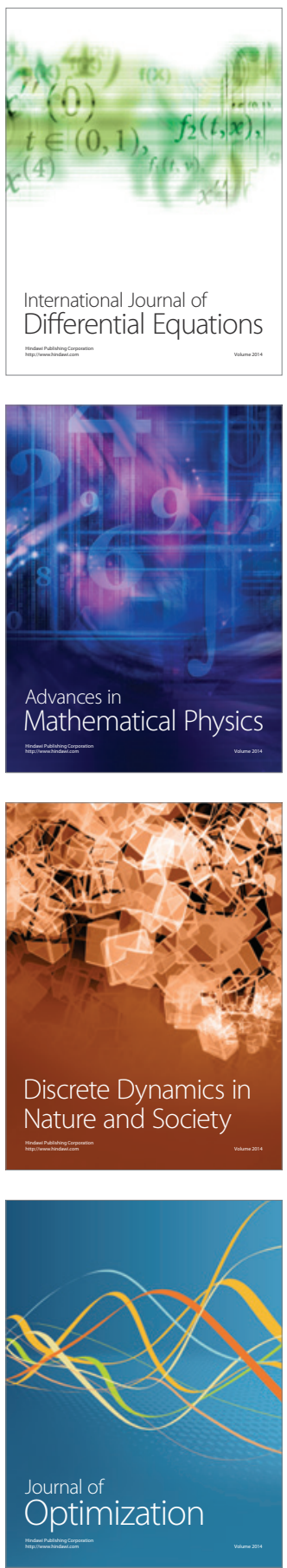